\title{
Neoadjuvant Therapy for Locally Advanced Renal Cell Carcinoma with Sorafenib in a Reference Center in Mexico
}

\author{
Axel Costilla-Montero ${ }^{1}$, Benjamín Guadarrama-Benítez ${ }^{1}$, Marco A. Aragón-Castro ${ }^{1}$, \\ Omar Morales-Ordaz ${ }^{1}$, Rubén Gutiérrez-Rosales ${ }^{1}$, Claudia Carrillo-Ponce ${ }^{2}$, \\ Marcela Janka-Zires ${ }^{2}$, Luis Gabriel Vázquez-Lavista ${ }^{{ }^{*}}$ \\ ${ }^{1}$ Departament of Urology, Centro Médico ISSEMYM Toluca, Toluca, México \\ ${ }^{2}$ Departament of Pathology, Centro Médico ISSEMYM Toluca, Toluca, México \\ ${ }^{3}$ Department of Internal Medicine Fundacion, Centro Médico ISSEMYM Toluca, Toluca, México \\ *E-mail: lugavala@yahoo.com \\ Received May 5, 2011; revised June 24, 2011; accepted July 7, 2011
}

\begin{abstract}
Background: neo-adjuvant therapy is usually indicated in locally advanced tumors, the aim is to decrease the tumoral burden and enhance overall survival. Renal cell carcinoma is a chemo and radio resistant neoplasm and this type of approach is not as effective as in other solid tumors. On the other hand immunotherapy is indicated in metastatic disease, demonstrating a better overall survival. Sorafenib is an antiangiogenic drug approved for locally advanced or metastatic RCC. We postulated that it can be used in a neoadjuvant way to decrease the vascularization of selected tumors. Report of the Case: 57 years old male referred to our service with a right renal mass and metastatic disease to lumbar spine and suprarenal gland. He was treated with three months of sorafenib previous to the surgery. Results: the patient went into surgery three months after initiating the antiangiogenic drug, during the surgery we found less neo-formance vessels; the dissection was subjectively easier, due to peri-renal edema. The pathologic analysis of the specimen was renal cell carcinoma. Interestingly, $40 \%$ of central ischemic (coagulative) necrosis was found. Conclusion: there are no neoadjuvant drugs accepted for the treatment of renal cell carcinoma; using an antiagiogenic drug to decrease the vascular burden characteristic of this type of tumors could be a viable option in selected cases. We used a lower dose of the drug with an acceptable safety profile.
\end{abstract}

Keywords: Renal Cell Carcinoma, Antiagiogenic Drugs, Neoadyvant Therapy, Sorafenib

\section{Introduction}

Kidney cancer is the seventh leading malignant condition among men and the twelfth among women, accounting for $2.6 \%$ of all cancers in the United States [1] and is the tenth cause in México [2]. The most frequent histology is renal clear cell carcinoma (RCCC). When RCCC in an organ confined stage, it is surgically treated with good overall survival rate, whoever, $30 \%$ of the cases are detected in an advanced stage, in the world and in México, with a high mortality rate $[3,4]$. RCCC shows poor response to chemotherapy due to multiple mechanisms [5]. Currently FDA approved treatment strategies for metastatic stages are immunotherapy with cytokines [interferon alpha and interleukine-2] and the use of antibodies against specific proteins (vascular endothelial growth factor, platelet derived growth factor, and tumor growth factor alpha) such as sunitinib [6], sorafenib [7] or bevacizumab [8].

Neo-adjuvant therapy is usually indicated in locally advanced tumors, and the aim is to decrease the tumoral burden and enhance overall survival. As mentioned above, due to the chemo-resitance of RCCC, neoadjuvant studies are scarce. On the other hand, the response to immunotherapy is limited and toxicicity is high. There are studies using immunotherapy as an adjuvant therapy along with cito-reductive nefrectomy with a better overall survival of the patients; [6-8] the aim of this study is to report our experience in one case treated with sorafenib as a neoadjuvant drug.

Scientific evidence suggests that the concept of 
neoadjuvant therapy for RCCC should be revisited [9], especially in selected patients according to the NCCN guidelines [10].

\section{Case Report}

Fifty seven year old male referred to our service with a right renal mass and metastatic disease to lumbar spine and suprarenal gland.

CT scan was performed with the evidence of a renal mass. He was treated with three months of sorafenib previous to the surgery. He received 200 mgs PO every 12 hrs for a month, the subsequent month no drug was administered, and the cycle was repeated for a third month. Figure 1 shows two slides of the CT scan demonstrating densities changes in the center of the mass before and after the neoadjuvant treatment.

\section{Results}

The patient went into surgery three months after initiating the antiangiogenic drug, during the surgery we found less neo-formance vessels; the dissection was subjectively easier, due to peri-renal edema. The pathologic analysis of the specimen was T4 renal cell carcinoma Fuhrman III in $95 \%$ of the kidney. Interestingly, $40 \%$ of central ischemic (coagulative) necrosis was found. The suprarenal gland was found with metastatic disease Figure 2.

\section{Discussion}

Controlled fase III studies have demonstrated superiority in those patients treated with INF and targeted therapies. Sorafenib is a multi kinase inhibitor including VEGFr,
PDGFr and RAF. Using it as a unique drug the results are promising, showing a better overall and progression free survival in metastatic disease [7]. One of the first case reports is bi Di Silverio et al. where they describe A 71-year-old man with advanced left renal cell carcinoma (lymph node involvement and vena cava thrombus) was submitted to 6 months of neoadjuvant treatment with sorafenib before open radical nephrectomy. After sorafenib treatment and before surgery a new CT scan confirmed the presence of a $9.0 \mathrm{~cm}$ in diameter solid mass in the left kidney but a reduction in thrombus extension, limited to the left renal vein. At histological examination after radical nephrectomy, over $90 \%$ of the renal mass was substituted by necrotic tissue [11].

Our patient showed 50\% necrosis in the pre surgery CT scans and this was corroborated in the final pathological specimen with $40 \%$ of central ischemic (coagulative) necrosis. It is worth to mention that the neoplastic necrosis is usually a liquefactive one, and it is characterized by digestion of the dead cells resulting in transformation of the tissues into a liquid viscous mass. In this case the architecture of the dead tissue was preserved and the affected tissue exhibited a firm texture so it was secondary to ischemia. Subjectively during the dissection we found a peri renal edema that made easier the surgery. It is also worth mentioning that the therapeutic dose used in this patient was lower than that used in a palliative scenery, and only a few adverse effects were reported, no important co morbidity was found during the follow up.

The biggest case control series with tyrosine kinase inhibition is with 14 patients who underwent cytoreductive nephrectomy for advanced renal cell carcinoma with prior treatment with sorafenib or sunitinib, and were compared with 73 patients without neoadjuvant therapy,

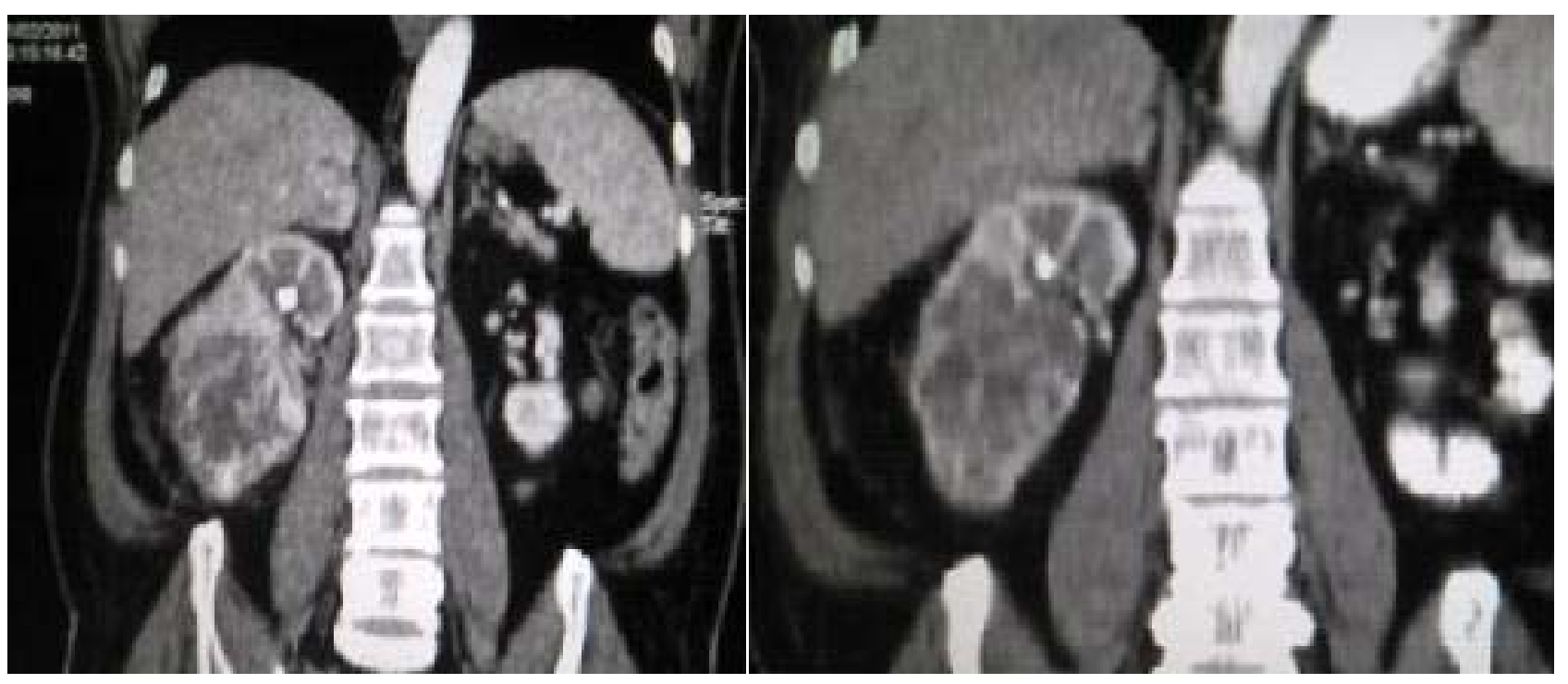

Figure 1. Pre- and post-neo-adjuvant treatment with sorafenib CT scan of the renal mass. 


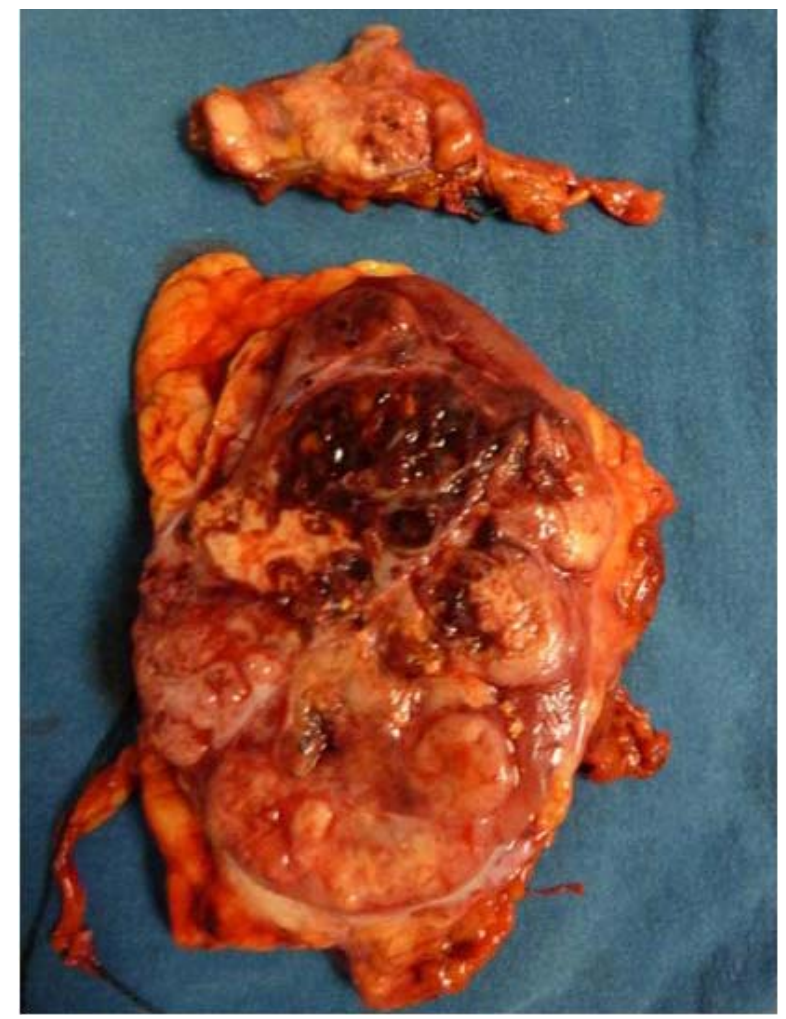

Figure 2. Pahtological specimen of right kidney with central necrosis and metastatic disease to suprarrenal gland.

the parameters studied were: the incidence of perioperative complications and outcomes after surgical procedures between the two cohorts. The median preoperative renal mass size was $11 \mathrm{~cm}(6.7-4.2 \mathrm{~cm})$. Primary tumor shrinkage was seen in 57\%; median shrinkage was $18 \%$ (8\% - 25\%). The median treatment period was 17 weeks, and the median time from TKI discontinuation was 2 weeks. Compared with a control group and after adjusting for confounding covariates, presurgical TKI use was not associated with a significant increase in perioperative complications ( $50 \%$ vs $40 \%, P=0.25$ ) or perioperative bleeding ( $36 \%$ vs $34 \%, P=0.97$ ) but was associated with increased incidence and grade of intraoperative adhesions ( $86 \%$ vs $58 \%, P=0.001$; grade 3 vs $1, P=$ 0.002). They concluded that they found less hemorrhagic and wound healing issues but a significant increase in incidence and severity of intraoperative adhesions, which can present a formidable technical challenge. The presurgical TKI therapy can permit effective surgical cytoreduction with a safety and complication profile equivalent to that of non-TKI-nephrectomy; however safety data continue to evolve, and preoperative TKI use requires further prospective investigation [12].

\section{Conclusions}

Sorafenib is a drug that inhibits specific proteins in neo- plastic cells, it modulates transduction signal and has shown to create intratumoral ischemic necrosis. The NCCN [9] and the EUA [13] guidelines have suggested that this drug can be used as a first line of treatment and the neoadjuvant setting should be investigated thoroughly.

\section{References}

[1] A. Jemal, T. Murray and E. Ward, "Cancer Statistics," CA: A Cancer Journal for Clinicians, Vol. 55, No. 1, 2005, pp. 10-30. doi:10.3322/canjclin.55.1.10

[2] Instituto Nacionall de Oncologia, "Manual de Oncologia Médica,” Procedimentos Medico Quirurgicos, 2 a Edición, McGraw Hill, Boston, 2002.

[3] L. M. Hock, J. Lynch and K. C. Balaji, "Increasing Incidences of all Stages of Kidney Cancer in the United States: An Analysis of Surveillance, Epidemiology and End Results Program Data," Journal of Urology, Vol. 167 , No. 1,2002 , pp. 57-60. doi:10.1016/S0022-5347(05)65382-7

[4] S. Ramsay and M. Aithkinson, "Treatment of Renal Cell Carcinoma: Are We beyond the Cytokine Era?” Nature Clinical Practice Urology, Vol. 3, No. 9, 2006, pp. 478-484. doi:10.1038/ncpuro0581

[5] E. Soto-Vega, C. Arroyo, Y. Richaud-Patin, M. García-Carrasco, L. G. Vázquez-Lavista and L. Llorente "PGlycoprotein Activity in Renal Clear Cell Carcinoma,” Urologic Oncology, Vol. 27, No. 4, 2009, pp. 363-366. doi:10.1016/j.urolonc.2008.01.011

[6] R. J. Motzer, T. E. Hutson, P. Tomczak, et al., "Sunitinib versus Interferon Alpha in Metastatci Renal Cell Carcinoma," The New England Journal of Medicine, Vol. 356, No. 2, 2007, pp. 115-124. doi:10.1056/NEJMoa065044

[7] B. Escudier, "Sorafenib in Advanced Clear-Cell Renal-Cell Carcinoma,” The New England Journal of Medicine, Vol. 356, No. 2, 2007, pp. 125-134. doi:10.1056/NEJMoa060655

[8] J. C. Young, L. Haworth, R. M. Sherry, et al., "A Randomized Trial of Bevacizumav an Antivascular Endothelial Growth Factor Antibody for Metastatic Renal Cell Cancer," The New England Journal of Medicine, Vol. 349, No. 5, 2003, pp. 427-434. doi:10.1056/NEJMoa021491

[9] C. Wood, et al., "Neoadjuvant (Presurgical) Therapy for Renal Cell Carcinoma: A New Treatment Paradigm for Locally Advanced and Metastatic Disease," Cancer, Vol. 115, No. S10, 2009, pp. 2355-2360. doi: $10.1002 /$ cncr. 24240

[10] National Comprehensive Cancer Network, "Clinical Practice Guidelines in Oncology ${ }^{\mathrm{TM}}$,” Kidney Cancer, V2, 2010. www.nccn.org

[11] F. Di siverio, A. Sciarra, U. Parente, A. Andrea, M. Von Heland, V. Panebianco and R. Passariello, "Neoadjuvant Therapy with Sorafenib in Advanced Renal Cell Carcinoma with Vena Cava Extension Submitted to Radical Nephrectomy,” Urologia Internationalis, Vol. 80, No. 4, 
2008, pp. 451-453.

[12] L. C. Harshman, R. J. Yu, G. I. Allen, S. Srinvas, H. S. Gill and B. I. Chung, "Surgical Outcomes and Complications Associated with Presurgical Tyrosine Kinase Inhibition for Advanced Renal Cell Carcinoma (RCC),”
Urologic Oncology, 24 February 2011.

[13] G. Mickisch, J. Carballido, S. Hellsten, H. Schulze, H. Mensink and European Association of Urology, "Guidelines on Renal Cell Cancer,” European Urology, Vol. 40, No. 3, 2001, pp. 252-255. doi:10.1159/000049783 